\title{
Fortaleciendo la transversalidad de los feminismos en la comunidad universitaria. La experiencia de la Facultad de Psicología de la Universidad Nacional de Córdoba, Argentina
}

\author{
Strengthening gender mainstreaming in the university community. The \\ experience of the Psychology College, National University of Cordoba, Argentina
}

\author{
Lorena Saletti-Cuesta \\ lorenasaletti@unc.edu.ar \\ Centro de Investigaciones y Estudios sobre Cultura y \\ Sociedad (UNC - CONICET). Universidad Nacional \\ de Córdoba, Argentina
}

Débora Imhoff

dimhoff@unc.edu.ar

Instituto de Investigaciones Psicológicas (UNC -

CONICET). Universidad Nacional de Córdoba,

Argentina

Recepción: 18 Mayo 2020

Aprobación: 18 Febrero 2021

Publicación: 01 Septiembre 2021

Cita sugerida: Saletti-Cuesta, L. y Imhoff, D. (2021).

Fortaleciendo la transversalidad de los feminismos en la comunidad universitaria. La experiencia de la Facultad de Psicología de la Universidad Nacional de Córdoba, Argentina. Descentrada, 5(2), e153. https://doi.org/10.24215/25457284e153
Resumen: Se detallan las acciones realizadas por la Prosecretaría de Género, Diversidad y Feminismos de la Facultad de Psicología (Universidad Nacional de Córdoba), las cuales se articulan en cuatro ejes de trabajo: 1) capacitación/sensibilización; 2) extensión; 3) abordaje de violencias de género en el ámbito universitario; 4) Facultad/Universidad inclusivas. Se presentan las acciones desarrolladas en el primer año de gestión (diciembre de 2018 a diciembre de 2019). Interesa compartir esta experiencia a fin de reflexionar sobre estas estrategias institucionales y la transversalización de las políticas y prácticas feministas dentro del ámbito de la universidad pública argentina.

Palabras clave: Transversalización de la perspectiva de género, Universidad, Políticas universitarias, Género, Feminismo.

\begin{abstract}
The actions carried out by the Pro-Secretary of Gender, Diversity and Feminism of the Psychology College (National University of Córdoba), are described and organized in four lines of work: (1) training/awareness; (2) extension; (3) addressing gender violence in the university environment; (4) inclusive College/university. The actions developed in the first year of management (December 2018 to December 2019) are presented. It is interesting to share this experience in order to reflect on these institutional strategies and the mainstreaming of feminist policies and practices within the scope of the Argentinian public university.
\end{abstract}

Keywords: Gender mainstreaming, University, University's politics, Gender, Feminist.

\section{INTRODUCCIÓN}

Las desigualdades articuladas al género adquieren un carácter de urgencia en nuestro contexto, también en las universidades. En las universidades argentinas, la presencia de las mujeres disminuye a mayor jerarquía del cargo (Secretaría de Políticas Universitarias, 2020). En el caso de las estudiantes, a pesar de que 
las mujeres son mayoría, están agrupadas en áreas consideradas tradicionalmente "femeninas" (Mollo Brisco y Moguiliansky, 2015; Agostini y Schiavi, 2017). Además de la segregación horizontal y vertical, diversas investigaciones señalan la existencia de violencias de género en nuestras universidades (Rovetto y Figueroa 2017; Rovetto, Figueroa, Montoni, y Fankhauser 2017; Carmody, Firpo y Genolet 2018; Vazquez Laba y Palumbo 2019; Soldevila y Domínguez, 2014; Rodigou Nocetti, Blanes, Burijovich y Domínguez 2011; Domínguez, Rodigou, Soldevila y Blanes 2018; Saletti-Cuesta, Quadri, Cordero, Scocozza y Bertarelli 2019; Saletti-Cuesta, 2019).

Los estudios feministas han demostrado que la presencia de las mujeres en las universidades, aun siendo en ocasiones mayoría, no es sinónimo de igualdad y que las desigualdades entre hombres y mujeres son producto de construcciones sociales, culturales e históricas, contribuyendo no sólo a visibilizar y estudiar esta problemática sino aportando estrategias para transformarla (Buquet Corleto, 2011).

Las universidades son espacios atravesados por las desigualdades de género, entre otras opresiones, con mecanismos y lógicas que las mantienen y las reproducen. $\mathrm{Al}$ mismo tiempo, son ámbitos de producción de saberes y acciones de transformación, e instituciones donde la sociedad deposita sus anhelos de cambio y justicia (Fabbri y Rovetto, 2020). En ese sentido, cada vez son más las universidades que implementan estrategias orientadas a construir una vida libre de discriminación y violencias. Por ejemplo, la aplicación de la Ley Nacional No 27.499 "Ley Micaela” en las Universidades Públicas, que establece la capacitación obligatoria en los temas de género y violencia contra las mujeres, o la implementación de protocolos para abordar las violencias de género. Resulta clave en el avance de estas políticas, el rol de la Red Interuniversitaria por la Igualdad de Género y Contra las Violencias (RUGE), creada en 2015 e incorporada al Consejo Interuniversitario Nacional (Rovetto, Figueroa, Montoni y Fankhauser, 2017). El impacto de estas estrategias excede el ámbito universitario y repercute en la construcción de equidad y justicia social para toda la sociedad (Buquet Corleto, 2011).

En tanto institución pública, la Universidad Nacional de Córdoba (UNC) ha asumido un fuerte compromiso con la promoción de acciones concretas, acorde con lo establecido por la normativa nacional e internacional vigente en nuestro país con relación a los Derechos Humanos y a las cuestiones de género. Como parte de ese compromiso, se creó en el año 2007 el Programa de Género de la Secretaría de Extensión de la UNC, elevada en 2019 al rango de Unidad Central de Políticas de Género, lo que implicó la modificación de la estructura orgánica del Rectorado y supuso la jerarquización institucional de este espacio en el ámbito universitario. En el año 2009 fue aprobada la Declaración de Derechos Estudiantiles, que expresamente establece el derecho a que la identidad y expresividad de género elegida y autopercibida sea reconocida en el ámbito universitario. En 2011, la Ordenanza No 9/11 declaró a la UNC como institución libre de discriminación por expresión o ejercicio de identidad de género. En el año 2012, la Ordenanza $\mathrm{N}^{\circ}$ 9/12, reglamentó el procedimiento para las investigaciones administrativas, incluyendo la discriminación y/o violencia de género. En el año 2015 el Consejo Superior aprobó el proyecto del Plan de acciones y herramientas para prevenir, atender y sancionar las violencias de género en el ámbito de la UNC (Res.HCSUNC 1011/2015).

En este marco de ampliación de derechos y de auge feminista en las universidades, a la vez que reconociendo las desigualdades de género en su propia comunidad, la Facultad de Psicología de la UNC asumió a fines de 2018 el compromiso de fortalecer el cambio cultural en su organización y funcionamiento. Así, en diciembre de 2018 se inauguró un nuevo espacio orientado al abordaje integral de estas problemáticas: la Prosecretaría de Género, Diversidad y Feminismos (GE.DI.FEM). Este espacio fue el primero de su rango en todo el conjunto de Facultades de la UNC.

El plan de gestión para el período 2018-2021 se articuló en función de cuatro ejes de trabajo: 1) Sensibilización y capacitación; 2) Facultad/Universidad inclusivas; 3) Abordaje de las violencias de género; 4) Extensión (hacia la comunidad universitaria y extrauniversitaria). 
A continuación, se presentan las acciones desarrolladas en el primer año de gestión (diciembre de 2018 a diciembre de 2019) en torno a cada uno de los ejes. Vale destacar que muchas de las actividades han sido coorganizadas con otras áreas de gestión de la Facultad, y/o de la UNC. Asimismo, todas han sido de carácter abierto y gratuito para los/as participantes y, en ocasiones, se otorgó certificación por la participación en las mismas como estrategia para la participación.

A su vez, de forma transversal al trabajo en cada uno de estos ejes, se difundió la Pro-secretaría a través de la web de la Facultad y sus redes oficiales. Se realizaron folletos explicativos, se organizaron reuniones con programas y servicios de extensión, y con equipos docentes. Se efectuaron presentaciones en Congresos, al tiempo que se participó activamente en las reuniones de la Mesa de áreas de Género de la UNC y en las de la Red Universitaria de Género (RUGE). También se redactaron informes de diversos aspectos solicitados oportunamente por el Consejo Directivo y el Superior. Asimismo, entre agosto de 2018 y noviembre de 2019, la Facultad de Psicología se abocó a un proceso colectivo de construcción de un nuevo Plan de Estudios para la Licenciatura en Psicología, y por ello varias de las actividades efectuadas por la Prosecretaría se enmarcaron en las iniciativas institucionales vinculadas con dicho proceso, promoviendo la discusión en torno a la formación desde una perspectiva de género. Como resultado, el 4 de noviembre de 2019 se aprobó el nuevo Plan de Estudios, que comprende como un eje nodal la inclusión transversal de contenidos de género en toda la currícula. Asimismo, en función de la adhesión de la UNC a la Ley Micaela, GE.DI.FEM participó activamente en el diseño de las capacitaciones virtuales.

En este trabajo, nos interesa describir las experiencias de la Prosecretaría y compartir el balance y reflexiones derivadas. Como indican Rovetto, Figueroa, Montoni y Fankhauser (2017), compartir las estrategias de incorporación de los feminismos en las universidades es importante para ponderar la relevancia de una política institucional situada, a la vez que ligada a una concepción integral de los feminismos en nuestra sociedad y, en especial, a la problemática de las violencias de género.

\section{EJE 1. ACCIONES DE SENSIBILIZACión Y CAPACITACIÓN EN TEMAS DE GÉNERO}

Si bien existen en nuestra comunidad personas que trabajan, investigan y ejercen docencia desde una perspectiva feminista desde hace tiempo, siendo su rol fundamental para la generación de conocimiento y prácticas críticas (Buquet Corleto, 2011), el abordaje sistemático de esta problemática en nuestra institución ha sido escaso. Por ello, para las acciones de este eje se seleccionaron en primer lugar temáticas de carácter introductorio. Se incorporaron instancias para vincular las perspectivas de género con la disciplina psicológica, al tiempo que se realizaron talleres y capacitaciones para articular el desarrollo de las tareas docentes y administrativas con esta mirada. A fines organizativos, se solicitó la inscripción de las personas interesadas en participar en las diversas acciones. Esta estrategia resultó interesante a la hora de conocer, por un lado, el grado de interés de la comunidad educativa en las temáticas propuestas; y por otro, el perfil de las personas participantes.

Se realizaron acciones destinadas a todos los claustros. Por ejemplo, la Prosecretaría dispone de un espacio de asesoramiento a demanda para la redacción no sexista de documentos y realiza talleres para nodocentes, estudiantes y docentes. Por otro lado, en el marco del 8 de Marzo se realizaron diversas actividades destinadas a toda la comunidad, como fueron la Ronda Debate "La violencia obstétrica también es violencia de Género"; el Panel "El ejercicio de la Psicología desde una perspectiva de género" y un taller de fanzine. En septiembre, en el marco de la firma de un convenio con la Asociación de Travestis, Transexuales y Transgéneros de Argentina (ATTTA), se realizaron los cursos de capacitación: "No estamos enfermas, estamos empoderadas", actividad sobre identidades trans desarrollada por integrantes de ATTTA, y la conferencia "Despatologizar el psicoanálisis de lo trans", actividad a cargo de una docente y estudiantes de nuestra Facultad. Asimismo, se realizaron diversos conversatorios abiertos a toda la comunidad universitaria y público en general: "Medios, ideología y patriarcado. Una crítica feminista a Game of Thrones"; "Experiencias de abortos inducidos en 
las trayectorias de vida de mujeres argentinas y mexicanas"; y "Sexología hoy: paradigmas en educación y diversidad sexual", entre otros.

Focalizándonos en el claustro estudiantil el 1 de marzo de 2019 se realizaron las III Jornadas de Género y Psicología, en las que se llevaron a cabo 40 talleres que abordaron distintas temáticas articuladas al género. Las jornadas estuvieron destinadas a ingresantes a la Facultad y tuvieron una alta concurrencia.

Dentro de las acciones destinadas a no docentes destacamos un taller sobre Violencias de género en la UNC y otra capacitación titulada "Introducción a la redacción no sexista". Asimismo, junto con el claustro docente, se difundió y promovió la participación en la Capacitación virtual en el marco de la aplicación de la Ley Micaela en la UNC.

Destinado tanto a no docentes como a docentes, se realizó el Curso virtual “Género, equidad y Educación Superior. Reflexiones y Prácticas Situadas”, a cargo de la Dra. Mercedes López. Este curso se propuso aportar a procesos de reflexión sobre temas vinculados con las desigualdades de género en la educación superior. Se realizaron dos ediciones, ambas con alta demanda y niveles de satisfacción.

En relación con el claustro docente las acciones estuvieron dirigidas al acompañamiento y a la capacitación en temas de género de cara al cambio de plan de estudios de la Facultad. Para ello se realizaron capacitaciones presenciales a cargo de especialistas en la temática. En marzo, se organizó el Taller de Actualización Docente: "Transformando las relaciones de género en el espacio universitario: El desafío de la inclusión sustantiva", cuyos objetivos se centraron en conocer y comprender los avances, retrocesos y desafíos para la inclusión de los estudios de género en los planes académicos de las instituciones de educación superior; analizar los problemas cotidianos que enfrentan el movimiento feminista y LGTTBQI para el acceso y permanencia de las mujeres y disidencias sexuales en las carreras de grado y posgrado, para la gestión de espacios de relevancia política y para la implementación de programas de inclusión y equidad de género, tomando como caso la UNC. También, con el fin de abordar los desafíos institucionales y personales para instalar las epistemologías, teorías y prácticas feministas en la UNC. En abril se colaboró y convocó a participar de la actividad organizada por el equipo de Investigación "Epistemologías, Teorías y Prácticas feministas por la Descolonización del conocimiento Hegemónico", ADIUC, el equipo de investigación El Telar: Comunidad de Pensamiento feminista Latinoamericano (FEMGES), la Facultad de Ciencias Sociales y el Programa Greda del CIECSCONICET-UNC. El encuentro se llamó "Feminismos y despatriarcalización" y sus objetivos fueron: generar una instancia de formación desde el cruce entre saberes académicos y comunitarios/territoriales; debatir el sentido de la formación y la extensión universitaria con toda la UNC y organizaciones sociales afines; y debatir cuáles son los desafíos del feminismo en el neoliberalismo actual y la potencia de los feminismos anticoloniales como teorías de emancipación, entre otros. En octubre, se realizó el Taller de Actualización Docente: "Ciencia, historia y feminismos: revisando la historia de la psicología", que se propuso promover una revisión crítica y feminista de la historia de la psicología para propiciar un debate en torno a la formación desde una perspectiva de género. Todos estos cursos fueron dictados por profesionales de amplia experiencia en el tema provenientes de instituciones y/u organizaciones externas a la Facultad de Psicología.

\section{EJE 2. FACUlTad/Universidad INCLUSIVAS}

Este eje busca promover diferentes iniciativas orientadas a transformar nuestra Facultad y Universidad en una comunidad inclusiva y sensible a las temáticas de género, y fomentar entre quienes integran nuestra comunidad educativa la incorporación de la perspectiva de género en los procesos y las rutinas institucionales. Este aspecto es impostergable para impulsar los cambios a través de las normas y reglamentos (Buquet Corleto, 2011), protagonizando así espacios estratégicos para transversalizar los feminismos como política institucional.

Para ello, en primer lugar, se revisaron las reglamentaciones de la Facultad a los fines de incorporar la perspectiva de género. En esa línea, en el primer semestre se presentó al Honorable Consejo Directivo 
una revisión del Reglamento Interno de Licencias Estudiantiles, sugiriendo la inclusión de una serie de modificaciones consonantes con una perspectiva de género que se constituyen en ampliatorias de derechos para las estudiantes mujeres y personas de otras identidades sexogenéricas. Tras su aprobación, se realizó una reunión informativa para toda la comunidad educativa en torno a la implementación del nuevo reglamento y se diseñó un vídeo explicativo (Facultad de Psicología, agosto 2019). En el segundo semestre, y a propuesta del claustro estudiantil, el Honorable Consejo Directivo de la Facultad de Psicología aprobó la utilización de lenguaje no sexista (RHCD N ${ }^{\circ}$ 347/2019). GE.DI.FEM tuvo un rol protagónico en el asesoramiento y retroalimentación del proyecto inicialmente presentado por el estudiantado, al tiempo que procedió a la difusión de la resolución aprobada.

Asimismo, GE.DI.FEM asesoró la presentación de propuestas de acción al Honorable Consejo Superior presentadas por la Decana de la Facultad. Se propuso un proyecto relativo a la reglamentación de los efectos de la renuncia cuando un/a agente se encuentre sometido/a a un proceso administrativo de investigación sumarial. El proyecto fue aprobado, emitiéndose resolución del HCS N 1795/2018 de diciembre de 2018. También se propuso la modificación del reglamento de concursos para incorporar en el art. 9 de la Ord. HCS 8/86 que podrán ser objeto de recusación los/as miembros/as del jurado y, por remisión del art. 13 de la misma Ordenanza, tener como causales de objeción de aspirantes inscriptos/as, a quienes hubieran incurrido en los supuestos contemplados como situaciones o casos de violencias de género, previstos en la RHCS 1011/2015. El proyecto se encuentra aún bajo tratamiento en el HCS al igual que el Proyecto de Protocolo de actuación para autoridades ante situaciones de violencia de género.

Otra de las acciones realizadas por GE.DI.FEM fue la orientación y acompañamiento a estudiantes trans que solicitaron cambio de nombre e identidad auto-percibida en los registros de la Facultad o que se encontraron con dificultades administrativas.

También se procedió a la reestructuración de espacios comunes a través de la instalación de cambiadores de bebés en los baños de mujeres, de varones y baño mixto para generar condiciones institucionales que acompañen una equidad de género en el cuidado de los/as hijos/as entre los/as miembros/as de nuestra comunidad educativa. Asimismo, con el objetivo de sensibilizar, visibilizar y conmemorar fechas claves se efectuaron comunicados que fueron difundidos por los medios oficiales de la Facultad.

\section{Eje 3. Abordaje de las violencias de género}

Sabemos que las violencias de género tienen un rol clave en la reproducción y el mantenimiento de las desigualdades de género (Saletti-Cuesta et al, 2019). Es por ello que se implementó un espacio específico de abordaje y asesoramiento de esta problemática en la Facultad.

Entre las actividades de difusión y sensibilización para la prevención de las violencias de género, se destacan la difusión del Plan de Acciones y Herramientas para prevenir, atender y sancionar las violencias de género (Res. HCS 1011/2015) y del espacio de atención en nuestra Facultad a toda la comunidad educativa a través de diversos medios (web institucional, redes sociales, Aula Virtual, mails, presentación en clases). Se difundió un curso virtual del Plan de Acciones y Herramientas para prevenir, atender y sancionar las violencias de género en la UNC destinado a estudiantes que consta de una fase virtual y de otra presencial para quienes tengan interés en formarse como promotores/as contra las violencias de género en la UNC.

Como acciones de sensibilización también se organizaron a lo largo del año cinco talleres: cuatro de ellos dirigidos a estudiantes, uno a no docentes y otro a toda la comunidad educativa. Este último fue un taller vivencial titulado "Herramientas para la prevención de las violencias de género desde la biodanza", donde se buscó sensibilizar sobre esta temática a través de técnicas vivenciales, trabajando especialmente la empatía, la escucha activa y el acompañamiento a estas problemáticas. En general, la participación en los talleres realizados por parte del claustro estudiantil fue baja. Por ello, y para fortalecer la sensibilización de este claustro, GE.DI.FEM diseñó el Curso virtual "Introducción a la problemática de las violencias de 
género". Se realizó en los meses de noviembre-diciembre de 2019, siendo su lanzamiento con ocasión del 25 de noviembre, Día Internacional de la Eliminación de la Violencia contra la Mujer. Sus contenidos se organizaron en tres unidades: difusión del Plan de Acciones y Herramientas para prevenir, atender y sancionar las violencias de género en la UNC, deconstrucción del amor romántico como un elemento clave en el mantenimiento de las violencias de género en el ámbito de las parejas, y masculinidades y violencias de género. El nivel de participación fue alto y la evaluación de los/as participantes fue muy positiva.

En cuanto al abordaje de situaciones de violencias de género, desde febrero hasta el 31 de diciembre, se atendieron un total de 32 consultas. La mayoría de ellas (20) fueron realizadas por el claustro estudiantil, en concordancia con las consultas del Equipo interdisciplinario del Plan de Acciones en la UNC (Saletti Cuesta, 2019). De las 32, solo cinco fueron realizadas por varones, el resto fueron llevadas a cabo por mujeres. Si bien algunas consultas atendidas se vincularon con situaciones fuera del ámbito universitario, la gran mayoría fueron de situaciones entre estudiantes, y de estudiantes con docentes de la casa. De las consultas provenientes de estudiantes, cuatro de ellas se vincularon con pedidos de licencia estudiantil por violencia de género en el marco del nuevo reglamento. Por otra parte, también se recibieron consultas de asesoramiento provenientes de otras instituciones y tres vinculadas al asesoramiento de prácticas supervisadas de fin de carrera relacionadas a la temática.

En relación con la atención de las situaciones de violencias de género, se realizaron numerosas articulaciones con diversas áreas de la Facultad y especialmente con el Plan de Acciones-UNC. Se efectuaron dos derivaciones a la atención psicológica del Plan de acciones y se acompañó y articuló la realización de dos manifiestos o pedidos de demanda. También se participó en todas las reuniones convocadas por el Plan de Acciones para articular la atención de las situaciones de violencias de género de los diversos espacios de la UNC. GE.DI.FEM acompañó fuertemente el proyecto del Plan de Acciones de formación de Promotores/ as de la UNC para la prevención de las violencias de género en la UNC "Estudiantes contra las violencias de género", abonando la implementación y construcción transversal de esta política en el marco de la universidad.

\section{Eje 4. EXTENSión}

Este eje busca colaborar al debate público a través de la generación de actividades dirigidas a la comunidad universitaria y, principalmente, extra-universitaria. Las actividades se articularon en tres grandes líneas: la promoción de las mujeres en la ciencia, la promoción de la igualdad de género en la infancia y acciones dirigidas a la población trans.

En cuanto a la primera, el 11 de febrero, Día Internacional de la Mujer y la Niña en la Ciencia, se realizó el I Encuentro de Mujeres en la Ciencia de la Facultad de Psicología, ocasión en la cual las científicas de nuestra institución se reunieron por primera vez para compartir experiencias en torno a los desafíos que implica desempeñarse en el mundo científico. Como resultado de dicho encuentro se redactó un documento público denunciando los numerosos obstáculos con los cuales se encuentran las científicas en el actual sistema científico nacional, fuertemente patriarcal y machista. El documento fue enviado a diversas agencias científicas y asociaciones. A su vez, en dicha ocasión se presentó públicamente un video institucional en el cual algunas de nuestras científicas nos contaron su experiencia como mujeres en la ciencia (Facultad de Psicología de la UNC, febrero 2019).

En relación a la promoción de la igualdad de género en la infancia, la Prosecretaría organizó a lo largo de todo el año el ciclo "Infancias Libres", que apuntó a la realización de actividades orientadas a fomentar una socialización con perspectiva de género durante la infancia, incluyendo para ello actividades recreativas y culturales con perspectiva de género para niños/as y talleres de capacitación para adultos/as que acompañan o trabajan con las infancias (progenitores/as, docentes, educadores/as no formales, etc.). El ciclo se inauguró 
el 9 de marzo de 2019 en el marco de las actividades previstas en la Facultad con ocasión del 8 de Marzo. Todas las actividades fueron abiertas y gratuitas.

En tercer lugar, en septiembre de 2019, y tras nueve meses de trabajo previo en torno al tema, se efectuó la firma de un convenio de trabajo y colaboración con ATTTA. La firma del convenio y todas las actividades desarrolladas en su marco se co-organizaron con la Subsecretaría de Servicios a la Comunidad de la Facultad de Psicología. Los objetivos generales del convenio fueron: 1) construir un vínculo interinstitucional de trabajo estable entre la Facultad de Psicología y ATTTA, que posibilite un abordaje de las problemáticas vinculadas con la población travesti y trans, así como la generación de iniciativas tendientes a promover un abordaje psicológico integral y acciones orientadas a la promoción de derechos en dicha población; 2) contribuir a promover el desarrollo integral de la población travesti y trans, 3) propiciar espacios de formación del estudiantado, de los claustros docente y no docente de la Facultad de Psicología en torno a las identidades trans desde una perspectiva de género y de derechos humanos. En ese marco, se realizaron las siguientes actividades:

- difusión del relevamiento online que realizó ATTTA, primer relevamiento de modalidad online dirigido hacia mujeres trans y travestis residentes de la ciudad de Córdoba.

- compra de material bibliográfico sobre identidades trans para incorporar al acervo de la biblioteca de la Facultad de Psicología.

- I Jornadas sobre identidades trans, con motivo de la firma del convenio. Las jornadas incluyeron la exposición de posters de trabajos de investigación y/o intervención con población travesti y trans en el hall de la Facultad de Psicología, la realización de una Mesa debate "Aportes desde la universidad pública al campo de lo trans", y el conversatorio titulado "Logros y desafíos actuales de la población trans".

- Programa de Asistencia Integral para Personas Travestis, Transexuales y Transgéneros. El Programa, de carácter gratuito, se encuentra coordinado por GE.DI.FEM y la Subsecretaría de Servicios a la Comunidad de la Facultad de Psicología. Incluye los siguientes servicios: 1) servicio de asistencia psicológica; 2) nodo de psicoanálisis aplicado a la demanda comunitaria; 3) prevención y asistencia neurocognitiva; 4) prevención y asistencia de consumos problemáticos; 5) servicio de consejerías en derechos sexuales, reproductivos y no-reproductivos; y 6) servicio de prevención y asistencia a parejas y familias. Se recibieron entre noviembre y diciembre de 2019 un total de siete consultas, cuatro de las cuales redundaron en el otorgamiento de turnos para atención psicológica en el marco del Programa.

\section{A MODO DE CIERRE}

Las acciones que estamos llevando adelante desde la Facultad de Psicología tienen la intención de efectuar un abordaje integral de las temáticas y problemáticas articuladas al género en nuestra institución. Enmarcadas en el compromiso que las universidades de nuestro país están adquiriendo en torno a estos temas, y en la potencia del movimiento feminista en nuestro contexto, GE.DI.FEM se inscribe en el convencimiento tanto del potencial de transformación feminista, como del rol crucial que las universidades públicas deben cumplir en los procesos de cambio cultural que nuestra sociedad requiere.

Consideramos que las actividades propuestas han permitido acercar esta mirada tanto a integrantes de nuestra comunidad educativa (de todos los claustros), como a la comunidad extra-universitaria. Se han realizado diversas instancias de capacitación y sensibilización, conversatorios, talleres vivenciales, cursos de capacitación presenciales y virtuales, talleres de capacitación docente, reuniones con equipos de cátedra, jornadas, rondas debate, paneles, talleres artísticos y culturales, actividades para niños/as y para adultos/ as que acompañan su crecimiento, y actividades con organizaciones sociales. También se promovió la revisión de reglamentaciones de la Facultad y de la Universidad desde una perspectiva de género, se 
propusieron reglamentaciones nuevas y se reestructuraron espacios comunes. Se efectuó un abordaje integral de las situaciones de violencia de género en el ámbito universitario, a través de un espacio de escucha, asesoramiento y acompañamiento y la realización de actividades de sensibilización. También se generaron acciones específicas para la población trans: acompañamiento a estudiantes, cursos y jornadas de formación, convenio con organizaciones trans, y la generación de un Programa de Atención Psicológica integral gratuito para población trans. Por último, se conmemoraron fechas claves del calendario feminista a través de comunicados públicos en redes sociales y medios oficiales de la Facultad, con el objetivo de fortalecer estos temas en la agenda universitaria.

En suma, todas las acciones desarrolladas han buscado promover, llevar de la periferia, de lo puntual y de las voluntades individuales, al centro, a la gestión de la política universitaria, la incorporación de la perspectiva de género en nuestra comunidad educativa. También, colaborar al debate público desde una mirada de género. En esa línea y a los fines de evaluar, al menos de algún modo, la implementación de estas políticas, se mantiene un registro sistemático de todas las acciones desarrolladas, con detalle del perfil de participantes de cada una, y -cuando ello resulta posible- se efectúa una evaluación cualitativa de las actividades por parte de los/as participantes tras su realización. Ello nos permite contar con herramientas que posibilitan una adecuada evaluación de las acciones propuestas, identificando los sectores de la comunidad educativa que se muestran más proclives a involucrarse en estos espacios y aquellos en los cuales aún persisten algunas resistencias. Asimismo, nos otorga elementos para evaluar las acciones desde la perspectiva de los/as propios/ as participantes. Por último, en 2020 se avanzó en una articulación con el Instituto de Investigaciones Psicológicas (IIPSI [CONICET y UNC]) a los fines de realizar un estudio longitudinal que permita identificar el impacto que las acciones desarrolladas por GE.DI.FEM posee en las trayectorias estudiantiles.

A modo de balance, consideramos que estas estrategias han sido positivas a pesar del gran número de desafíos aún por enfrentar, especialmente para transversalizar los feminismos y permear toda la vida universitaria. Como ejemplo de algunos puntos a trabajar podemos mencionar, por un lado, la necesidad de fortalecer la sensibilización entre el claustro estudiantil, ya que su participación en la mayoría de las actividades ha sido baja, y por otro, la importancia trabajar en la prevención integral de las violencias de género y avanzar hacia la transformación de los vínculos. Asimismo, consideramos que existe un gran interés en nuestra comunidad educativa por los estudios de género: hay cada vez más tesis de grado y postgrado, curso, graduados/as y docentes con trayectoria en estos temas. Sin embargo, la articulación de GE.DI.FEM con este cuerpo formado y su participación en las estrategias implementadas es aún un desafío a trabajar que, sin duda, contribuiría a fortalecer la incorporación de esta perspectiva en la formación académica y en el diseño y evaluación de acciones. El camino emprendido no es fácil, pero apostamos a transformar colectivamente el status quo de género presente en nuestra Facultad con un compromiso ético y político feminista.

\section{REFERENCIAS}

Agostini, A. y Schiavi, N. (2017). La presencia de las mujeres en la Universidad y su participación politica en órganos de gestión y representación. Recuperado de Observatorio de Derechos Humanos de la Universidad Nacional de Córdoba: https://www.unc.edu.ar/sites/default/files/Informe\%20final.\%20Observatorio\%20DDHH.UNC_ .pdf

Buquet Corleto, A.G. (2011). Transversalización de la perspectiva de género en la educación superior: Problemas conceptuales y prácticos. Perfiles educativos, 33, 211-225.

Carmody, C., Firpo, I., y Genolet, A. (2018). Apuntes sobre el enfoque de género en la UNER: entre lo construido y lo pendiente para su consolidación En: Rojo, P. y Jardon, V. (Comp.) Los enfoques de género en las Universidades (pp. 41-53.) Rosario: Universidad Nacional de Rosario, Asoc. Universidades Grupo Montevideo.

Domínguez, A., Rodigou, M., Soldevila, A., y Blanes, P. (2018). Investigar para Transformar: desafíos pendientes en torno a las Violencias de Género en la Universidad. En: Rojo, P. y Jardon, V. (Comp.) Los enfoques de género 
en las Universidades (pp, 122-137). Rosario: Universidad Nacional de Rosario, Asoc. Universidades Grupo Montevideo.

Fabbri, L., y Rovetto, F. (2020). Introducción Cuadernos feministas para la transversalización. En Attardo, CI. et al. Apuntes sobre género en curriculas e investigación. (pp. 9-16). Rosario: Editorial de la Universidad Nacional de Rosario. Recuperado de: http://ruge.cin.edu.ar/attachments/article/24/04714_02\%20-\%20Apuntes\%20sobre \%20g\%C3\%A9nero\%20en\%20curr\%C3\%ADculas\%20e\%20investigaci\%C3\%B3n.pdf

Facultad de Psicología. Universidad Nacional de Córdoba. (febrero, 2019). Día de la niña y la mujer científica [video en YouTube]. Recuperado de https://www.youtube.com/watch?v=X757Zfem4o8\&t=1s

Facultad de Psicología. Universidad Nacional de Córdoba. (2009) Plan de Estudios de la Carrera de Psicología RD $\mathrm{N}^{\circ}$ 2165/2019. Recuperado de: http://www.digesto.unc.edu.ar/psicologia/decanato/resolucion/2165_2019/? searchterm $=2165$

Facultad de Psicología. Universidad Nacional de Córdoba. (agosto, 2019). Nuevo reglamento de licencias estudiantiles [video en YouTube]. Recuperado de https://www.youtube.com/watch?v=gf6nQXeOHNY

Mollo Brisco, G. y Moguiliansky, M.S. (2015). Mujeres Directivas en las Universidades Nacionales Argentinas. Ciencias Administrativas, 3(5), 39-51. Recuperado de https://revistas.unlp.edu.ar/CADM/article/view/880

Rodigou Nocetti, M., Blanes, P., Burijovich, J., y Domínguez, A. (2011). Trabajar en la Universidad: (Des) Igualdades de género por transformar. Córdoba: Universidad Nacional de Córdoba.

Rovetto, F. y Figueroa, N. (2017). "Que la universidad se pinte de feminismo" para enfrentar las violencias sexistas. Descentrada, 1(2). Recuperado de: https://www.descentrada.fahce.unlp.edu.ar/article/view/DESe026/8707

Rovetto, F., Figueroa, N., Montoni, R. y Fankhauser, I. (2017). La desnaturalización de las violencias sexistas en las Universidades. Una experiencia de visibilización y prevención en la Universidad Nacional de Rosario. Revista Ensambles, 4(7), 83-98.

Saletti-Cuesta, L., Quadri, S., Cordero, M.L., Scocozza, R. y Bertarelli, P. (2019). Desafíos para abordar las situaciones de violencia de género en el ámbito universitario de Córdoba (Argentina).Rued@. Revista Universidad, Ética y Derecho, 3-4, 126-135.

Saletti-Cuesta, L. (editora) (2019). Plan de acciones y herramientas para prevenir, atender y sancionar las violencias de género en la UNC. Informe de gestión 2017-2018. Córdoba: Universidad Nacional de Córdoba. Recuperado de: http://www.fcq.unc.edu.ar/sites/default/files/pictures/informe_2017-18.pdf

Secretaría de Políticas Universitarias (2020). Mujeres en el Sistema Universitario Argentino 2018-2019. Recuperado de: https://www.argentina.gob.ar/sites/default/files/mujeres_en_el_sistema_universitario_argentino_-_estadi sticas_2018-2019.pdf

Soldevila, A. y Domínguez, A. (2014). Violencia de género, una realidad en la universidad. Córdoba: Universidad Nacional de Córdoba.

Vázquez Laba, V. y Palumbo, M. (2019). Causas y efectos de la discriminación y la violencia de género en el ámbito universitario. Descentrada, 3(2), e093. https://doi.org/10.24215/25457284e093 